\title{
Phase II trial of bevacizumab and erlotinib as a second-line therapy for advanced hepatocellular carcinoma
}

\author{
Ahmed O Kaseb ${ }^{1}$ \\ Jeffrey S Morris ${ }^{2}$ \\ Michiko Iwasaki' \\ Humaid O Al-Shamsi' \\ Kanwal Pratap Singh \\ Raghav' \\ Lauren Girard' \\ Sheree Cheung' \\ Van Nguyen ${ }^{3}$ \\ Khaled M Elsayes ${ }^{4}$ \\ Lianchun $\mathrm{Xiao}^{2}$ \\ Reham Abdel-Wahab ${ }^{1,5}$ \\ Ahmed S Shalaby' \\ Manal Hassan' \\ Hesham M Hassabo' \\ Robert A Wolff' \\ James $C$ Yao' \\ 'Department of Gastrointestinal \\ Medical Oncology, ${ }^{2}$ Department \\ of Biostatistics, ${ }^{3}$ Department of \\ Pharmacy, ${ }^{4}$ Department of Diagnostic \\ Radiology, The University of Texas MD \\ Anderson Cancer Center, Houston, \\ TX, USA; ${ }^{5}$ Department of Clinical \\ Oncology, Assiut University Hospital, \\ Assiut, Egypt
}

Correspondence: Ahmed Omar Kaseb Department of Gastrointestinal Medical Oncology, Unit 426, The University of Texas MD Anderson Cancer Center, I5I 5 Holcombe Blvd, Houston, TX 77030, USA

$\mathrm{Tel}+\mathrm{I} 7 \mid 37922828$

Fax + I 713745 II63

Email akaseb@mdanderson.org
This article was published in the following Dove Press journal:

OncoTargets and Therapy

15 February 2016

Number of times this article has been viewed

Trial registry: Clinicaltrials.gov \#NCT01180959.

Background: Early clinical studies of bevacizumab and erlotinib in advanced hepatocellular carcinoma (HCC) have a tolerable toxicity and a promising clinical outcome. We evaluated the efficacy and tolerability of this combination as a second-line therapy for HCC refractory to sorafenib.

Methods: For this single-arm, Phase II study, we recruited patients with Child-Pugh class A or B liver disease, Eastern Cooperative Oncology Group performance status 0-2, and advanced HCC that was not amenable to surgical or regional therapies and treatment with sorafenib had failed (disease progressed or patient could not tolerate sorafenib). Patients received $10 \mathrm{mg} / \mathrm{kg}$ intravenous bevacizumab every 14 days and $150 \mathrm{mg}$ oral erlotinib daily for 28-day cycles until progression. Tumor response was evaluated every two cycles using Response Evaluation Criteria in Solid Tumors. The primary end point was the 16-week progression-free survival rate. Secondary end points included time to progression and overall survival.

Results: A total of 44 patients were enrolled and had a median follow-up time of 33.8 months (95\% confidence interval [CI]: 23.5 months - not defined). The 16-week progression-free survival rate was $43 \%$ (95\% CI: $28 \%-59 \%)$, median time to progression was 3.9 months (95\% CI: $2.0-8.3$ months), and median overall survival duration was 9.9 months (95\% CI: 8.3-15.5 months). Grade 3-4 adverse events included fatigue (13\%), acne (11\%), diarrhea (9\%), anemia (7\%), and upper gastrointestinal hemorrhage (7\%).

Conclusion: Bevacizumab plus erlotinib was tolerable and showed a signal of survival benefit in the second-line setting for patients with advanced HCC. Because standard-of-care options are lacking in this setting, further studies to identify predictors of response to this regimen are warranted.

Keywords: hepatocellular carcinoma, targeted therapy, bevacizumab, erlotinib, second-line therapy

\section{Introduction}

Hepatocellular carcinoma (HCC) is the fifth most common malignancy worldwide and the third leading cause of cancer-related mortality, and HCC is now the leading cause of death in patients with cirrhosis. ${ }^{1}$ In the USA, HCC is the fastest growing cause of cancer-related mortality in American men. ${ }^{2}$ Notably, poor outcomes with HCC are mainly due to late stages of disease at diagnosis and lack of effective treatments. Approximately $80 \%$ of HCC patients are initially diagnosed with advanced-stage disease with underlying cirrhosis, which precludes curative treatment options such as surgical resection or liver transplantation. Sorafenib is the only approved systemic therapy for advanced HCC, but it has shown only modest activity in randomized Phase 
III trials. ${ }^{3,4}$ Therefore, patients eventually develop either progressive disease or intolerance to sorafenib, presenting a major challenge that warrants the development of secondline therapies for HCC.

Given that $\mathrm{HCC}$ is an angiogenesis-driven tumor, agents targeting angiogenesis have been investigated, with unsatisfactory results. ${ }^{5-7}$ Our group and others have reported promising activity in HCC for the combination of bevacizumab, an intravenous monoclonal antibody that binds circulating vascular endothelial growth factor-A ligand, and erlotinib, an oral tyrosine kinase inhibitor that inhibits phosphorylation of the intracellular domain of the epidermal growth factor receptor (EGFR). Notably, several studies showed overexpression of EGFR in HCC, ${ }^{8,9}$ and the potential involvement of EGFR in angiogenesis. ${ }^{10}$

Recent Phase II HCC studies, including two studies in the first-line setting conducted by our group, indicated that this combination showed activity in HCC. ${ }^{11-16}$ However, variable survival outcomes suggested potential differential effects with patient demographics, geographic location, HCC risk factors, and tumor and cirrhosis grade. Furthermore, to date, no Phase II studies of this combination in the secondline setting have been reported in the US patients with HCC. Thus, currently, there are no approved systemic therapies for patients with advanced HCC that progressed in response to sorafenib.

We hypothesized that the combination of bevacizumab plus erlotinib is a viable second-line therapy for advanced HCC in which treatment with sorafenib has failed. The primary end point of the current study was the 16-week progression-free survival (PFS) rate, and secondary end points included time to progression (TTP), overall survival (OS), and rates of adverse events.

\section{Methods}

We conducted a Phase II, single-arm, open-label study of bevacizumab plus erlotinib as a second-line therapy for advanced HCC in which first-line treatment with sorafenib failed owing to disease progression or drug intolerance. The study was approved by the Institutional Review Board of The University of Texas MD Anderson Cancer Center and was conducted in accordance with the principles of the Declaration of Helsinki. All patients provided written informed consent.

\section{Eligibility criteria}

We recruited patients aged 18 years or older who had advanced HCC that were not amenable to surgical or regional therapies and for which prior treatment with sorafenib had failed. All patients had measurable disease as per modified
Response Evaluation Criteria in Solid Tumors. Prior nonsystemic treatments such as surgical resection, transarterial embolization or chemoembolization, radiofrequency ablation, or percutaneous ethanol injection were allowed, provided that evaluable lesions were separate from previously treated lesions. Other inclusion criteria were Eastern Cooperative Oncology Group performance status of $\leq 2$; Child-Pugh liver function class A or B (with a score of up to 7 points), absolute neutrophil count $\geq 1,500 \mathrm{~mm}^{3}$, platelet count $\geq 40,000 \mathrm{~mm}^{3}$, hemoglobin level $\geq 10 \mathrm{~g} / \mathrm{dL}$, total bilirubin level $\leq 2.0 \mathrm{~g} / \mathrm{dL}$, serum albumin level $\geq 2.5 \mathrm{~g} / \mathrm{dL}$, aspartate aminotransferase and alanine aminotransferase levels up to five times the upper limit of institutional normal (46 IU/L for aspartate aminotransferase and $56 \mathrm{IU} / \mathrm{L}$ for alanine aminotransferase), and prothrombin time no more than 3 seconds longer than institutional normal.

\section{Treatment schedule}

All patients received a 30 -minute infusion of $10 \mathrm{mg} / \mathrm{kg}$ bevacizumab intravenously once every 2 weeks and $150 \mathrm{mg}$ of erlotinib orally once daily on an outpatient basis. Cycles were repeated every 28 days. Erlotinib was self-administered in an open-label, unblinded manner to all patients enrolled in the study. Treatment was continued until disease progression, occurrence of an unacceptable adverse event, or patient withdrawal of consent. Treatment modifications were permitted according to study protocol.

\section{Disease assessment}

Each patient's medical history, Eastern Cooperative Oncology Group performance status, physical examination findings, laboratory results, and adverse events were recorded at the start of every cycle. Restaging evaluation was performed after every two cycles. Tumor response was evaluated using modified Response Evaluation Criteria in Solid Tumors criteria. ${ }^{17}$ Evaluable patients included any patient who received at least one 28-day cycle. Patients were observed until death or until a maximum of 3 years after enrollment. All tumor measurements were obtained by the investigator and were reviewed by a collaborating diagnostic radiologist.

\section{Statistical methods}

The primary end point was the 16-week PFS rate. PFS was defined as the time from initiation of therapy until documented disease progression or death. The proportion of patients who were progression-free at 16 weeks was estimated with a $95 \%$ confidence interval (CI) and tested using a one-sided binomial exact test. Because objective tumor responses are not expected with the use of biologic agents 
in HCC, the combination of bevacizumab plus erlotinib was considered potentially active, if the 16-week PFS rate was similar to that of historic controls. ${ }^{13}$

The secondary end point of TTP was defined as the time between treatment assignment and radiologic progression. Other secondary end points included response rate (complete and partial response); median OS duration; safety; toxicity and tolerability; stable disease at 16, 24, and 36 weeks; and duration of response.

A one-sided binomial exact test was applied to compare the success rate with the null hypothesis; Simon's two-stage optimal design was implemented. Specifically, we used the optimal two-stage design to test the null hypothesis of 16-week PFS success rate $P_{0} \leq 0.350$ compared with the alternative of $P_{1} \geq 0.550$ with $80 \%$ power at a one-sided alpha level of 0.05 . With this design, if 20 or fewer patients out of 44 were alive and progression-free at 16 weeks, the drug would be considered not effective. To evaluate predictors of outcome (OS and PFS), we initially used Cox proportional hazards regression models and log-rank tests to uncover associations between patient clinicopathologic characteristics and OS or PFS. Then, multivariate Cox models of variables with $P<0.1$ in univariate analysis were fitted to identify independent prognostic factors, and the backward selection procedure was used for model selection. All data were analyzed using SAS (SAS Institute, Cary, NC, USA) and Splus software v8.2 (TIBCO, Palo Alto, CA, USA), and $P \leq 0.05$ was considered statistically significant.

\section{Results}

\section{Patient characteristics}

A total of 44 patients were enrolled and received at least one dose of bevacizumab plus erlotinib and were included in the final analysis, they had the median follow-up time of 33.8 months (95\% CI: 23.5 months - not defined). Table 1 shows the baseline characteristics of these patients. The median age at baseline was 63 years; 33 patients $(75 \%)$ were male and eleven patients (25\%) were female; 19 patients $(43 \%)$ had pathologic or radiographic evidence of cirrhosis; 26 patients (59\%) were white; 43 patients (98\%) had Child-Pugh class A liver function; and 34 patients (77\%) had a Cancer of the Liver Italian Program (CLIP) score of 0-2 and seven patients (16\%) had a CLIP score of 3-4. The CLIP score, a prognostic system that has been prospectively validated in several studies, assigns points for the following: Child-Pugh score, tumor morphology (solitary, $\leq 50 \%$ of the liver, massive), serum $\alpha$-fetoprotein level, and presence or absence of portal vein thrombus. ${ }^{18}$ Almost all of the patients $(41 ; 93 \%)$ had a stage $C$ tumor according to the
Table I Baseline characteristics of patients included in our final analysis $(n=44)$

\begin{tabular}{|c|c|}
\hline Variable & $\mathbf{N}$ \\
\hline Age (median \pm SD [range]), years & $63.05 \pm$ I I.46 (32-82) \\
\hline \multicolumn{2}{|l|}{ Sex } \\
\hline Female & II (25) \\
\hline Male & $33(75)$ \\
\hline \multicolumn{2}{|c|}{ Reason for ceasing previous treatment with sorafenib } \\
\hline Mean duration of treatment \pm & $7.53 \pm 10.31(0.5-60)$ \\
\hline \multicolumn{2}{|l|}{ SD (range), months } \\
\hline Progressive disease & $33(75)$ \\
\hline Progressive disease and toxic effects & $4(9)$ \\
\hline Toxic effects & $7(16)$ \\
\hline Cirrhosis & $19(43)$ \\
\hline Hepatitis $C$ virus & $13(30)$ \\
\hline Hepatitis $B$ virus & $8(18)$ \\
\hline Nonalcoholic fatty liver disease & $0(0)$ \\
\hline Alcoholism & $10(23)$ \\
\hline Diabetes mellitus & $16(36)$ \\
\hline Metabolic syndrome & $8(18)$ \\
\hline Follicular nodular hyperplasia & I (2) \\
\hline \multicolumn{2}{|l|}{ ECOG performance status } \\
\hline 0 & $15(34)$ \\
\hline 1 & $29(66)$ \\
\hline BMI (median \pm SD [range]), kg/m² & $28.2 \pm 6.52(18.6-49.5)$ \\
\hline \multicolumn{2}{|l|}{ Ethnicity } \\
\hline Asian & $3(7)$ \\
\hline African American & $5(I I)$ \\
\hline White & $26(59)$ \\
\hline Hispanic & $9(20)$ \\
\hline Unknown & I (2) \\
\hline \multicolumn{2}{|l|}{$\alpha$-fetoprotein level } \\
\hline$\leq 400$ & $32(73)$ \\
\hline$>400$ & $12(27)$ \\
\hline \multicolumn{2}{|l|}{ Child-Pugh class } \\
\hline$A$ & $43(98)$ \\
\hline B & I (2) \\
\hline \multicolumn{2}{|l|}{ Albumin level } \\
\hline $2.5-2.8 \mathrm{~g} / \mathrm{dL}$ & I (2) \\
\hline $2.8-3.5 \mathrm{~g} / \mathrm{dL}$ & $8(18)$ \\
\hline$>3.5 \mathrm{~g} / \mathrm{dL}$ & $35(80)$ \\
\hline \multicolumn{2}{|l|}{ Tumor volume } \\
\hline$>50 \%$ & $9(20)$ \\
\hline$\leq 50 \%$ & $32(73)$ \\
\hline Unknown & $3(7)$ \\
\hline \multicolumn{2}{|l|}{ Tumor morphology } \\
\hline Multicellular & $34(77)$ \\
\hline Unicellular & $7(16)$ \\
\hline Free & $3(7)$ \\
\hline \multicolumn{2}{|l|}{ Barcelona Clinic Liver Cancer stage } \\
\hline$A$ & $2(5)$ \\
\hline B & I (2) \\
\hline C & $4 I(93)$ \\
\hline \multicolumn{2}{|l|}{ Cancer of the Liver Italian Program score } \\
\hline 0 & $5(I I)$ \\
\hline 1 & $17(39)$ \\
\hline 2 & $12(27)$ \\
\hline 3 & $7(16)$ \\
\hline 4 & 0 \\
\hline Unknown & $3(7)$ \\
\hline
\end{tabular}


Table I (Continued)

\begin{tabular}{ll}
\hline Variable & N \\
\hline American Joint Committee on Cancer TNM stage & \\
II & $7(16)$ \\
IIIA & $3(7)$ \\
IIIB & $5(11)$ \\
IV & $29(66)$ \\
Okuda stage & \\
0 & $27(6 \mathrm{I})$ \\
I & $13(30)$ \\
2 & $1(2)$ \\
Unknown & $3(7)$ \\
\hline
\end{tabular}

Note: Data presented as $\mathrm{N}$ (\%), unless otherwise stated.

Abbreviations: BMI, body mass index; ECOG, Eastern Cooperative Oncology Group; SD, standard deviation.

Barcelona Clinic Liver Cancer staging classification. ${ }^{19}$ The mean ( \pm standard deviation) baseline $\alpha$-fetoprotein level was $4,918.98 \pm 16,077.99 \mathrm{ng} / \mathrm{mL}$ and the mean baseline hemoglobin level was $12.51 \pm 1.46 \mathrm{~g} / \mathrm{dL}$.

\section{Response and survival}

The 16-week PFS rate was 43\% (95\% CI: 28\%-59\%); 19 of 44 patients were alive and progression-free at 16 weeks. This percentage was not significantly greater than the null hypothesized value of $35 \%$ of patients (one-sided binomial exact test, $P=0.16)$. At 16 weeks, 4 patients $(9 \%)$ achieved partial response, 18 patients (41\%) had stable disease, 4 patients (9\%) had progressive disease, and 3 patients (7\%) were not evaluable for response evaluation. The median TTP was 3.9 months (95\% CI: $2.0-8.3$ months) and the median OS duration was 9.9 months (95\% CI: 8.3-15.5 months).

\section{Clinical predictors of outcome}

Table 2 shows the results of the log-rank test to evaluate associations between baseline categorical clinical predictors and OS or PFS. The analysis showed a significant association between tumor volume and $\operatorname{OS}(P=0.0128)$ and tumor morphology and OS $(P=0.0291)$. Patients with a tumor volume of $>50 \%$ had shorter OS durations than those with a tumor volume of $\leq 50 \%$, and patients with multicellular tumor morphology had shorter OS durations than those with unicellular tumor morphology. The log-rank test also showed that focal nodular hyperplasia $(P<0.0001)$, tumor volume $>50 \%(P=0.002)$, multicellular tumor morphology $(P=0.0042)$, and lymph node metastasis $(P=0.0353)$ were associated with reduced PFS duration.

Results of the univariate Cox proportional hazards regression analyses of associations between baseline continuous variables and OS and PFS are shown in Tables 3 (OS) and 4 (PFS), respectively. Low alkaline phosphatase levels (hazard ratio [HR]: $1.0020,95 \% \mathrm{CI}: 1.000-1.003, P=0.0476)$, small tumors (HR: $1.1220,95 \% \mathrm{CI}: 1.021-1.232, P=0.0160)$, and high hemoglobin levels (HR: 0.6958, 95\% CI: 0.538-0.8991, $P=0.0055)$ were associated with increased OS duration in the univariate analysis. In addition to low alkaline phosphatase levels and high hemoglobin levels, age and low $\alpha$-fetoprotein levels were associated with increased PFS duration (Table 4).

The multivariate Cox proportional hazards regression analysis indicated that hepatitis B virus infection was associated with decreased OS duration (HR: 2.27, 95\% CI: $1-5.14, P=0.05)$ and high hemoglobin levels were associated with increased OS duration (HR: 0.69, 95\% CI: 0.53-0.89, $P=0.0046$ ). Similarly, the multivariate Cox analysis for PFS showed that hepatitis $\mathrm{C}$ virus infection (HR: $0.32,95 \% \mathrm{CI}$ : 0.14-0.76, $P=0.009$ ), age (HR: 0.96, 95\% CI: 0.92-0.99, $P=0.01$ ), and high hemoglobin levels (HR: 0.69, 95\% CI: 0.53-0.89, $P=0.0039)$ were associated with increased PFS duration. However, high $\alpha$-fetoprotein levels (HR: 1.00003, 95\% CI: $1.00002-1.00005, P=0.02$ ) were associated with decreased PFS duration.

\section{Toxicity and dose modification}

All patients were assessed for adverse events, which are shown in Table 5. Grade 3 or 4 adverse events included fatigue $(n=6 ; 13 \%)$, acne $(n=5 ; 11 \%)$, diarrhea $(n=4 ; 9 \%)$, anemia $(n=3 ; 7 \%)$, upper gastrointestinal hemorrhage $(n=3$; $7 \%)$, elevated transaminases $(n=1)$, hyperbilirubinemia $(n=1)$, wound infection $(n=1)$, hand-foot syndrome $(n=1)$, nausea $(n=1)$, anorexia $(n=1)$, constipation $(n=1)$, mucositis $(n=1)$, hypertension $(n=1)$, syncope attack $(n=1)$, and thrombus formation $(n=1)$. At the time of enrollment, patients with a history of substantial gastrointestinal bleeding requiring procedural intervention within 3 months prior to treatment day 1 and patients at risk for varices (on the basis of known history of esophageal or gastric varices or evidence of hepatic cirrhosis or portal hypertension, including biopsy-proven cirrhosis, radiographic evidence of cirrhosis, hypersplenism, or radiographic findings of varices) were screened for esophageal varices. Patients requiring intervention for varices did not receive treatment with bevacizumab plus erlotinib until the varices were treated.

\section{Discussion}

Despite not meeting the primary end point, we believe that our study results demonstrate that the combination of bevacizumab plus erlotinib is safe and possesses a signal of survival benefit per our secondary end points and therefore 
Table 2 Baseline clinical predictors of OS and PFS according to the log-rank test

\begin{tabular}{|c|c|c|c|c|}
\hline \multirow[t]{2}{*}{ Clinical variable } & \multicolumn{2}{|l|}{ PFS } & \multicolumn{2}{|l|}{ OS } \\
\hline & Median PFS (95\% Cl), months & $P$-value & Median OS $(95 \% \mathrm{Cl})$, months & $P$-value \\
\hline Acneiform rash & & 0.2436 & & 0.1945 \\
\hline No $(n=9)$ & $2.14(1.81$ to $N D)$ & & 7.4 (5.03 to ND) & \\
\hline Yes $(n=35)$ & $5.69(2.04-13.45)$ & & $10.33(9.24-17.2)$ & \\
\hline Cirrhosis & & 0.0763 & & - \\
\hline Absent $(n=25)$ & $2.14(1.81-7.89)$ & & $9.8(7.4-14.67)$ & \\
\hline Present $(n=19)$ & 7.4 (4.3I to ND) & & II.6I (8.09 to ND) & \\
\hline $\mathrm{HCV}$ status & & 0.0701 & & 0.2835 \\
\hline Negative $(n=3 \mid)$ & $2.83(1.91-7.89)$ & & $9.8(8.09-\mid 4.67)$ & \\
\hline Positive $(n=13)$ & $8.52(1.91$ to ND) & & 11.61 (5.82 to ND) & \\
\hline HBV status & & 0.0701 & & 0.0573 \\
\hline Negative $(n=36)$ & $5.02(2.14-10.33)$ & & $10.33(8.32-16.58)$ & \\
\hline Positive $(n=8)$ & $1.92(1.81$ to ND) & & 8.26 (5.53 to ND) & \\
\hline CLIP score & & 0.5824 & & 0.5939 \\
\hline $0-2(n=35)$ & $5.02(2.14-13.45)$ & & $11.05(9.24-17.2)$ & \\
\hline $3-4(n=9)$ & 3.65 (I.78 to ND) & & 9.31 (7.27 to ND) & \\
\hline Child-Pugh class & & 0.6768 & & 0.0219 \\
\hline$A(n=43)$ & $4.31(2.04-8.52)$ & & $10.07(8.32-15.49)$ & \\
\hline$B(n=I)$ & $3.65(\mathrm{ND})$ & & $5.03(\mathrm{ND})$ & \\
\hline $\mathrm{FNH}$ & & $<0.0001$ & & 0.2132 \\
\hline No $(n=43)$ & 4.31 (2.04-8.52) & & | $0.07(8.32-\mid 5.49)$ & \\
\hline Yes $(n=I)$ & 1.32 (ND) & & $6.88(N D)$ & \\
\hline Tumor volume $\mathrm{e}^{\mathrm{a}}$ & & 0.002 & & 0.0128 \\
\hline$>50 \%(n=9)$ & 2.04 (I.8I to ND) & & 8.32 (7.4 to ND) & \\
\hline$\leq 50 \%(n=32)$ & $7.34(4.31-16.58)$ & & $13.42(9.5|-2| .74)$ & \\
\hline Tumor morphology & & 0.0042 & & 0.0291 \\
\hline Multicellular $(n=34)$ & 3.01 (I.9I-7.27) & & $10.07(9.24-\mid 5.49)$ & \\
\hline Unicellular $(n=7)$ & 16.18 (8.32 to ND) & & 16.18 (8.32 to ND) & \\
\hline Free $(n=3)$ & 1.68 (I.32 to ND) & & 6.41 (5.82 to ND) & \\
\hline BCLC stage & & 0.0693 & & 0.0235 \\
\hline$A(n=2)$ & ND (ND) & & ND (ND) & \\
\hline$B(n=I)$ & 1.81 (ND) & & $5.03(\mathrm{ND})$ & \\
\hline$C(n=4 I)$ & $3.65(2.04-7.89)$ & & $9.8(8.32-14.67)$ & \\
\hline Okuda stage ${ }^{\mathrm{a}}$ & & 0.0208 & & 0.0057 \\
\hline $0(n=27)$ & $7.89(4.31-17.3)$ & & $15.49(10.33-28.72)$ & \\
\hline I $(n=13)$ & 2.14 (I.91 to ND) & & 8.09 (6.84 to ND) & \\
\hline $2(n=1)$ & 8.32 (ND) & & 8.32 (ND) & \\
\hline
\end{tabular}

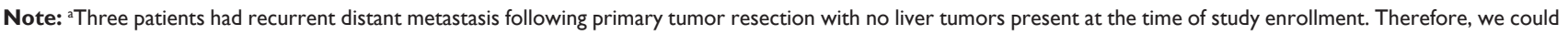
not calculate percentage of liver involvement or OKUDA stage.

Abbreviations: $\mathrm{Cl}$, confidence interval; PFS, progression-free survival; OS, overall survival; ND, not defined; HBV, hepatitis B virus; HCV, hepatitis C virus; CLIP, Cancer of the Liver Italian Program; FNH, follicular nodular hyperplasia; BCLC, Barcelona Clinic Liver Cancer.

could be a promising regimen for select patients with advanced HCC refractory to sorafenib.

The primary objective was to assess the 16-week PFS rate, which was $43 \%$ with a one-sided $95 \%$ CI. Although

Table 3 Univariate Cox proportional hazards regression model of associations between overall survival and baseline continuous variables

\begin{tabular}{lll}
\hline Covariate & HR $(95 \% \mathrm{CI})$ & $\boldsymbol{P}$-value \\
\hline Age at the time of inclusion & $0.98(0.96-1.01)$ & 0.34 \\
Alkaline phosphatase levels & $1.00(1.000-1.003)$ & 0.04 \\
$\alpha$-fetoprotein levels & $1.00(1.00-1.00)$ & 0.43 \\
Tumor size & $1.12(1.02-1.23)$ & 0.01 \\
Hemoglobin levels & $0.69(0.53-0.89)$ & 0.005 \\
\hline
\end{tabular}

Abbreviations: $\mathrm{HR}$, hazard ratio; $\mathrm{Cl}$, confidence interval. this is greater than the $35 \%$ historic value, we could not reject the null hypothesis, which stipulated a $45 \%$ cutoff at the one-sided significance level of 0.05 to be considered successful. However, the survival outcome of our study

Table 4 Univariate Cox proportional hazards regression model of associations between progression-free survival and baseline continuous variables

\begin{tabular}{lll}
\hline Covariate & HR (95\% CI) & P-value \\
\hline Age at the time of inclusion & $0.96(0.93-0.99)$ & 0.04 \\
Alkaline phosphatase levels & $1.00(1.000-1.004)$ & 0.02 \\
$\alpha$-fetoprotein levels & $1.00(1.00-1.00)$ & 0.00 \\
Tumor size & $1.06(0.98-1.16)$ & 0.12 \\
Hemoglobin levels & $0.73(0.57-0.95)$ & 0.01 \\
\hline
\end{tabular}

Abbreviations: $\mathrm{HR}$, hazard ratio; $\mathrm{Cl}$, confidence interval. 
Table 5 Adverse events $(n=44)$ by toxicity grade

\begin{tabular}{|c|c|c|c|}
\hline \multirow[t]{2}{*}{ Adverse event } & \multicolumn{3}{|c|}{ Toxicity grade, $\mathbf{N}(\%)$} \\
\hline & $I-2$ & 3 & 4 \\
\hline \multicolumn{4}{|l|}{ Constitutional events } \\
\hline Fever without neutropenia & I (2) & $0(0)$ & $0(0)$ \\
\hline Fatigue & $23(5 \mathrm{I})$ & $6(13)$ & $0(0)$ \\
\hline Weight loss & $10(22)$ & $0(0)$ & $0(0)$ \\
\hline Dyspnea & $3(7)$ & $0(0)$ & $0(0)$ \\
\hline Other & $6(13)$ & $0(0)$ & $0(0)$ \\
\hline \multicolumn{4}{|l|}{ Gastrointestinal events } \\
\hline Anorexia & $20(44)$ & I (2) & $0(0)$ \\
\hline Nausea & $16(36)$ & I (2) & $0(0)$ \\
\hline Vomiting & $13(29)$ & $0(0)$ & $0(0)$ \\
\hline Dysphagia & $2(4)$ & $0(0)$ & $0(0)$ \\
\hline Diarrhea & $22(49)$ & $4(9)$ & $0(0)$ \\
\hline Constipation & $4(9)$ & I (2) & $0(0)$ \\
\hline Other & $6(13)$ & $0(0)$ & $0(0)$ \\
\hline \multicolumn{4}{|l|}{ Dermatologic events } \\
\hline Acne & $40(89)$ & $5(\mathrm{II})$ & $0(0)$ \\
\hline Alopecia & I (2) & $0(0)$ & $0(0)$ \\
\hline Dry skin & $24(53)$ & $0(0)$ & $0(0)$ \\
\hline Pruritus & $4(9)$ & $0(0)$ & $0(0)$ \\
\hline Rash & $3(7)$ & $0(0)$ & $0(0)$ \\
\hline Bruising & I (2) & $0(0)$ & $0(0)$ \\
\hline \multicolumn{4}{|l|}{ Gastrointestinal hemorrhage } \\
\hline Lower gastrointestinal tract & $6(13)$ & I (2) & $0(0)$ \\
\hline Upper gastrointestinal tract & I (2) & $3(7)$ & $0(0)$ \\
\hline Nose bleeding & $10(22)$ & $0(0)$ & $0(0)$ \\
\hline \multicolumn{4}{|l|}{ Pain } \\
\hline Head & $7(156)$ & $0(0)$ & $0(0)$ \\
\hline Back & $2(4)$ & $0(0)$ & $0(0)$ \\
\hline Abdomen & $4(9)$ & $0(0)$ & $0(0)$ \\
\hline Muscle & $2(4)$ & I (2) & $0(0)$ \\
\hline Other & $10(22)$ & $I(2)$ & $0(0)$ \\
\hline Anemia & $3(7)$ & $3(7)$ & $0(0)$ \\
\hline Elevated transaminases & $5(\mathrm{II})$ & I (2) & $0(0)$ \\
\hline Hand-foot syndrome & $3(7)$ & $\mathrm{I}(2)$ & $0(0)$ \\
\hline Wound infection & I (2) & $0(0)$ & $0(0)$ \\
\hline Pulmonary hemorrhage & $3(7)$ & $0(0)$ & $0(0)$ \\
\hline Hyperbilirubinemia & $13(29)$ & I (2) & $0(0)$ \\
\hline Hypokalemia & $3(7)$ & $0(0)$ & $0(0)$ \\
\hline Dry mouth & $2(4)$ & $0(0)$ & $0(0)$ \\
\hline Dry eyes & $\mathrm{I}(2)$ & $0(0)$ & $0(0)$ \\
\hline Hypertension & $7(16)$ & I (2) & $0(0)$ \\
\hline Hyperpigmentation & I (2) & $0(0)$ & $0(0)$ \\
\hline Hypomagnesemia & $7(16)$ & $0(0)$ & $0(0)$ \\
\hline Nail changes & $4(9)$ & $0(0)$ & $0(0)$ \\
\hline Proteinuria & $18(40)$ & $0(0)$ & $0(0)$ \\
\hline Mucositis & $25(556)$ & I (2) & $0(0)$ \\
\hline Taste alteration & $7(16)$ & $0(0)$ & $0(0)$ \\
\hline Voice changes & $7(16)$ & $0(0)$ & $0(0)$ \\
\hline Infection & $4(9)$ & I (2) & I (2) \\
\hline Syncope attacks & $0(0)$ & I (2) & $0(0)$ \\
\hline Thrombus formation & I (2) & I (2) & $0(0)$ \\
\hline
\end{tabular}

looks promising as compared with failed recent randomized second-line studies in HCC. Furthermore, this could be related to the inherit challenges of Phase II studies, including under-power and false-negative rates. Therefore, the survival signal of our combination should be investigated in future studies in HCC patients in the second-line treatment setting in the USA, focusing on biomarker studies that could help select potential subset of patients who may benefit from this combination. Such studies are critically important, given the lack of any standard-of-care options for HCC in the second-line treatment setting. Our recent frontline HCC study of bevacizumab and erlotinib showed a significant correlation between higher plasma level of angiopoietin 2 and shorter OS. ${ }^{13}$ Other studies reported the potential prognostic value of plasma expression of Ang-2, ${ }^{20-25}$ and the potential association with resistance to anti-angiogenesis therapy. ${ }^{18,26}$ Furthermore, immunohistochemistry assay of EGFR from prior HCC studies did not show a correlation between EGFR expression and treatment outcomes. ${ }^{8,9}$ However, in our more recent study, ${ }^{13}$ lower plasma level of EGFR was marginally associated with resistance to therapy as defined by tumor progression $(P=0.076)$. Therefore, further studies to identify predictors of response to this regimen are warranted.

Our findings indicate that the combination of bevacizumab plus erlotinib was tolerated and showed some activity, as demonstrated by the median TTP of 3.9 months and median OS duration of 9.9 months. This is the second study to examine the combination of bevacizumab plus erlotinib in sorafenib-refractory disease and the only such study in the US patients. Yau et a ${ }^{15}$ conducted a small second-line Phase II study in patients from Asia with sorafenib-refractory HCC, but that study was halted after enrolling ten patients owing to lack of efficacy, with a reported TTP of 1.81 months $(95 \% \mathrm{CI}$ : $1.08-1.74$ months) and median OS duration of 4.37 months (95\% CI: $1.08-11.66$ months). Yau et a ${ }^{15}$ reported that rash $(70 \%)$, diarrhea $(50 \%)$, and malaise $(40 \%)$ were the most commonly encountered toxic effects, and they concluded that the combination of bevacizumab plus erlotinib was well tolerated but had no activity in an unselected population with sorafenib-refractory advanced HCC.

Our study is distinct from this prior study in that the prior study was conducted in an Asian population, in which the incidence of hepatitis B virus infection was higher than in Western populations. ${ }^{19}$ Evidently, $82 \%$ of patients in that study had hepatitis B virus-associated HCC, whereas only $18 \%$ of patients in our study had a hepatitis B virus infection. It is quite clear from analysis of sorafenib trials that patient demographics and the etiology of HCC may be responsible, at least partially, for differential outcomes in patients with HCC; therefore, a dedicated study in a US population is needed to establish the efficacy of the bevacizumab plus erlotinib regimen. $., 27,28$

These observations concerning geographic location of the study population are consistent with data from prior pivotal 
studies of local and systemic therapies for HCC and highlight the need to assess and compare results from HCC clinical trials in the context of geographic location, demographics, and HCC risk factors. For example, a Phase III study of sorafenib (Sorafenib HCC Assessment Randomized Protocol) ${ }^{3}$ reported a median OS duration of 10.7 months in the sorafenib arm and 7.9 months in the placebo $\operatorname{arm}(P<0.001)$ and a median TTP of 5.5 months in the sorafenib arm and 2.8 months in the placebo arm $(P<0.001)$. An Asia-Pacific Phase III sorafenib study also reported improvement in the median OS duration (6.5 months compared with 4.2 months; $P=0.014$ ) and TTP (2.8 months compared with 1.4 months; $P=0.0005$ ) with sorafenib compared with placebo, but the magnitude of the improvement was marginal. In addition, further subgroup analyses of data from sorafenib Phase III studies also showed differential outcomes in patients based on demographics and hepatitis status. ${ }^{27}$

Notably, multiple studies have evaluated the role of bevacizumab plus erlotinib in the treatment of $\mathrm{HCC}$ in the first-line setting. The observed 16-week PFS rate in our current second-line study was lower than in our earlier first-line trials of this combination in patients with advanced HCC. Thomas et $\mathrm{al}^{16}$ reported a 16 -week PFS rate of $62.5 \%$ and Kaseb et $\mathrm{al}^{13}$ reported a rate of $64 \%$. However, in a similar study of 51 Asian patients with advanced untreated HCC, Hsu et al ${ }^{12}$ reported a 16 -week PFS rate of only $35.3 \%$. In another Phase II study of 27 patients with advanced HCC, Philip et $\mathrm{al}^{14}$ reported a partial response in one patient and stable disease in eleven patients (48\%). The median TTP was 3.0 months (95\% CI: 1.8-7.1 months) and the median OS duration was 9.5 months (95\% CI: 7.1-17.1 months). However, one major limitation of this US study was that the primary end point was objective response rate, which is an inadequate surrogate marker for response in HCC in the context of targeted therapy. Govindarajan et $\mathrm{al}^{11}$ also reported a nonsignificant 27-week PFS rate of 28\% among a group of 20 patients with HCC, who were not candidates for local therapy. The rate is lower than that of the historic control arm of the Sorafenib HCC Assessment Randomized Protocol trial. The authors suggested that lack of administration of any prior therapy with radiofrequency ablation or transarterial chemoembolization could have led to inferior results because these procedures are known to improve survival outcomes.

The adverse event rates in the current study were similar to those reported in earlier trials. ${ }^{13,16}$ The dose was not modified in any of the patients in our study, although one patient withdrew consent after receiving two cycles. Most of the patients had Barcelona Clinic Liver Cancer stage $\mathrm{C}$ disease and a CLIP score of 2 or 3 , and the median age of the population was older than 60 years. Our results showed that clinical predictors such as Child-Pugh class B, age, low baseline hemoglobin levels, high baseline $\alpha$-fetoprotein levels, high baseline alkaline phosphatase levels, tumor volume $\geq 50 \%$, presence of lymphatic metastasis, multicellular tumor morphology, CLIP score $>3$, and Okuda stage $\geq 2$ were indicators of poor prognosis in HCC.

As in other single-arm, Phase II trials, the limitations of this study include single-institution enrollment, small sample size, lack of a control arm, and potential for institutional and investigator selection bias. In addition, patients referred to a tertiary care cancer center may represent a patient population with more favorable outcomes than would otherwise be expected, particularly in $\mathrm{HCC}$.

In conclusion, in this first US study of bevacizumab plus erlotinib as second-line therapy after progression on sorafenib, the treatment combination appears to be safe and shows interesting activity in advanced HCC. Our study findings are relevant to the US patients, given the recent failure of multiple other systemic therapies in this setting, especially in light of the tolerability of the combination, and given that the natural history of $\mathrm{HCC}$ and treatment outcomes differ by patient demographics, geographic location, and $\mathrm{HCC}$ risk factors. ${ }^{28}$

\section{Acknowledgment}

This study was supported by Roche and Genentech Inc.

\section{Disclosure}

The authors report no conflicts of interest in this work.

\section{References}

1. Llovet JM, Burroughs A, Bruix J. Hepatocellular carcinoma. Lancet. 2003;362(9399):1907-1917.

2. El-Serag HB, Rudolph KL. Hepatocellular carcinoma: epidemiology and molecular carcinogenesis. Gastroenterology. 2007;132(7): 2557-2576.

3. Llovet JM, Ricci S, Mazzaferro V, et al. Sorafenib in advanced hepatocellular carcinoma. N Engl J Med. 2008;359(4):378-390.

4. Cheng AL, Kang YK, Chen Z, et al. Efficacy and safety of sorafenib in patients in the Asia-Pacific region with advanced hepatocellular carcinoma: a phase III randomised, double-blind, placebo-controlled trial. Lancet Oncol. 2009;10(1):25-34.

5. Finn RS, Kang YK, Mulcahy M, et al. Phase II, open-label study of brivanib as second-line therapy in patients with advanced hepatocellular carcinoma. Clin Cancer Res. 2012;18(7):2090-2098.

6. Zhu AX, Kudo M, Assenat E, et al. EVOLVE-1: Phase 3 study of everolimus for advanced HCC that progressed during or after sorafenib. J Clin Oncol. 2014;32(Suppl 3):abstr 172.

7. Zhu AX, Ryoo B-Y, Yen C-J, et al. Ramucirumab (RAM) as secondline treatment in patients (pts) with advanced hepatocellular carcinoma (HCC): analysis of patients with elevated $\alpha$-fetoprotein (AFP) from the randomized phase III REACH study. J Clin Oncol. 2015;33(Suppl 3): abstr 232 . 
8. Fausto N. Growth factors in liver development, regeneration and carcinogenesis. Prog Growth Factor Res. 1991;3(3):219-234.

9. Hisaka T, Yano H, Haramaki M, Utsunomiya I, Kojiro M. Expressions of epidermal growth factor family and its receptor in hepatocellular carcinoma cell lines: relationship to cell proliferation. Int J Oncol. 1999;14(3): 453-460.

10. Hirata A, Ogawa S, Kometani T, et al. ZD1839 (Iressa) induces antiangiogenic effects through inhibition of epidermal growth factor receptor tyrosine kinase. Cancer Res. 2002;62(9):2554-2560.

11. Govindarajan R, Siegel E, Makhoul I, Williamson S. Bevacizumab and erlotinib in previously untreated inoperable and metastatic hepatocellular carcinoma. Am J Clin Oncol. 2013;36(3):254-257.

12. Hsu CH, Kang YK, Yang TS, et al. Bevacizumab with erlotinib as firstline therapy in Asian patients with advanced hepatocellular carcinoma: a multicenter phase II study. Oncology. 2013;85(1):44-52.

13. Kaseb AO, Garrett-Mayer E, Morris JS, et al. Efficacy of bevacizumab plus erlotinib for advanced hepatocellular carcinoma and predictors of outcome: final results of a phase II trial. Oncology. 2012;82(2):67-74.

14. Philip PA, Mahoney MR, Holen KD, et al. Phase 2 study of bevacizumab plus erlotinib in patients with advanced hepatocellular cancer. Cancer. 2012;118(9):2424-2430.

15. Yau T, Wong H, Chan P, et al. Phase II study of bevacizumab and erlotinib in the treatment of advanced hepatocellular carcinoma patients with sorafenib-refractory disease. Invest New Drugs. 2012;30(6): 2384-2390.

16. Thomas MB, Morris JS, Chadha R, et al. Phase II trial of the combination of bevacizumab and erlotinib in patients who have advanced hepatocellular carcinoma. J Clin Oncol. 2009;27(6):843-850.

17. Therasse P, Arbuck SG, Eisenhauer EA, et al. New guidelines to evaluate the response to treatment in solid tumors. European Organization for Research and Treatment of Cancer, National Cancer Institute of the United States, National Cancer Institute of Canada. J Natl Cancer Inst. 2000;92(3):205-216.

18. Llovet J, Pena C, Shan M, Lathia C, Bruix J. Plasma biomarkers as predictors of outcome in patients with advanced hepatocellular carcinoma: results from the randomized phase III trial. EASL Annual Meeting; 2009.
19. Park JW, Chen M, Colombo M, et al. Global patterns of hepatocellular carcinoma management from diagnosis to death: the BRIDGE Study. Liver Int. 2015;35(9):2155-2166.

20. Scholz A, Rehm VA, Rieke S, et al. Angiopoietin-2 serum levels are elevated in patients with liver cirrhosis and hepatocellular carcinoma. Am J Gastroenterol. 2007;102(11):2471-2481.

21. Torimura T, Ueno T, Kin M, et al. Overexpression of angiopoietin-1 and angiopoietin-2 in hepatocellular carcinoma. $J$ Hepatol. 2004; 40(5):799-807.

22. Moon WS, Rhyu KH, Kang MJ, et al. Overexpression of VEGF and angiopoietin 2: a key to high vascularity of hepatocellular carcinoma? Mod Pathol. 2003;16(6):552-557.

23. Tanaka S, Mori M, Sakamoto Y, Makuuchi MN, Sugimachi K, Wands JR. Biologic significance of angiopoietin-2 expression in human hepatocellular carcinoma. J Clin Invest. 1999;103(3):341-345.

24. Wada H, Nagano H, Yamamoto H, et al. Expression pattern of angiogenic factors and prognosis after hepatic resection in hepatocellular carcinoma: importance of angiopoietin-2 and hypoxia-induced factor-1 alpha. Liver Int. 2006;26(4):414-423.

25. Chen ZB, Shen SQ, Ding YM, et al. The angiogenic and prognostic implications of VEGF, Ang-1, Ang-2, and MMP-9 for hepatocellular carcinoma with background of hepatitis B virus. Med Oncol. 2009; 26(3):365-371

26. Reig ME, Rodriguez de Lope C, Forner A, et al. Impact of early treatment interruption and biomarker profiling in patients with $\mathrm{HCC}$ treated with sorafenib. The Liver Meeting 2010 (AASLD), Abstract No 1748.

27. Bruix J, Raoul JL, Sherman M, et al. Efficacy and safety of sorafenib in patients with advanced hepatocellular carcinoma: subanalyses of a phase III trial. J Hepatol. 2012;57(4):821-829.

28. Kaseb AO. Beyond the basics: the differential effects of demographics and hepatitis status on treatment outcome in hepatocellular carcinoma. Oncology. 2013;85(1):41-43.
OncoTargets and Therapy

\section{Publish your work in this journal}

OncoTargets and Therapy is an international, peer-reviewed, open access journal focusing on the pathological basis of all cancers, potential targets for therapy and treatment protocols employed to improve the management of cancer patients. The journal also focuses on the impact of management programs and new therapeutic agents and protocols on

\section{Dovepress}

patient perspectives such as quality of life, adherence and satisfaction The manuscript management system is completely online and includes a very quick and fair peer-review system, which is all easy to use. Visit http://www.dovepress.com/testimonials.php to read real quotes from published authors. 